\title{
ASO Visual Abstract: Outcomes of Sentinel Node Biopsy in Women with Breast Cancer Following Neoadjuvant Therapy- Systematic Review and Meta-Analysis of Real-World Data
}

\begin{abstract}
Shi-Qian Lin, MS ${ }^{1}$, Nguyen-Phong Vo, $\mathrm{MD}^{2}$, Yu-Chun Yen, $\mathrm{MS}^{3}$, and Ka-Wai Tam, MD, $\mathbf{P h D}^{4,5,6,7}$
${ }^{1}$ School of Medicine, College of Medicine, Taipei Medical University, Taipei, Taiwan; ${ }^{2}$ International PhD Program in Medicine, College of Medicine, Taipei Medical University, Taipei, Taiwan; ${ }^{3}$ Biostatistics Center, Office of Data Science, Taipei Medical University, Taipei, Taiwan; ${ }^{4}$ Division of General Surgery, Department of Surgery, Shuang Ho Hospital, Taipei Medical University, New Taipei City, Taiwan; ${ }^{5}$ Division of General Surgery, Department of Surgery, School of Medicine, College of Medicine, Taipei Medical University, Taipei, Taiwan; ${ }^{6}$ Cochrane Taiwan, Taipei Medical University, Taipei, Taiwan; ${ }^{7}$ Center for Evidence-Based Health Care, Shuang Ho Hospital, Taipei Medical University, New Taipei City, Taiwan
\end{abstract}

The accuracy of sentinel lymph node biopsy (SLNB) following neoadjuvant therapy (NAT) in patients with breast cancer is inconclusive. Current real-world evidence revealed that the false negative rate (FNR) of SLNB and identification rate of sentinel lymph nodes (SLNs) after
NAT were $11.8 \%$ and $90.6 \%$, respectively. Patients with estrogen receptor (ER)-negative status and fewer than three SLNs removed had a significantly higher FNR (https://doi. org/10.1245/s10434-021-11297-z).

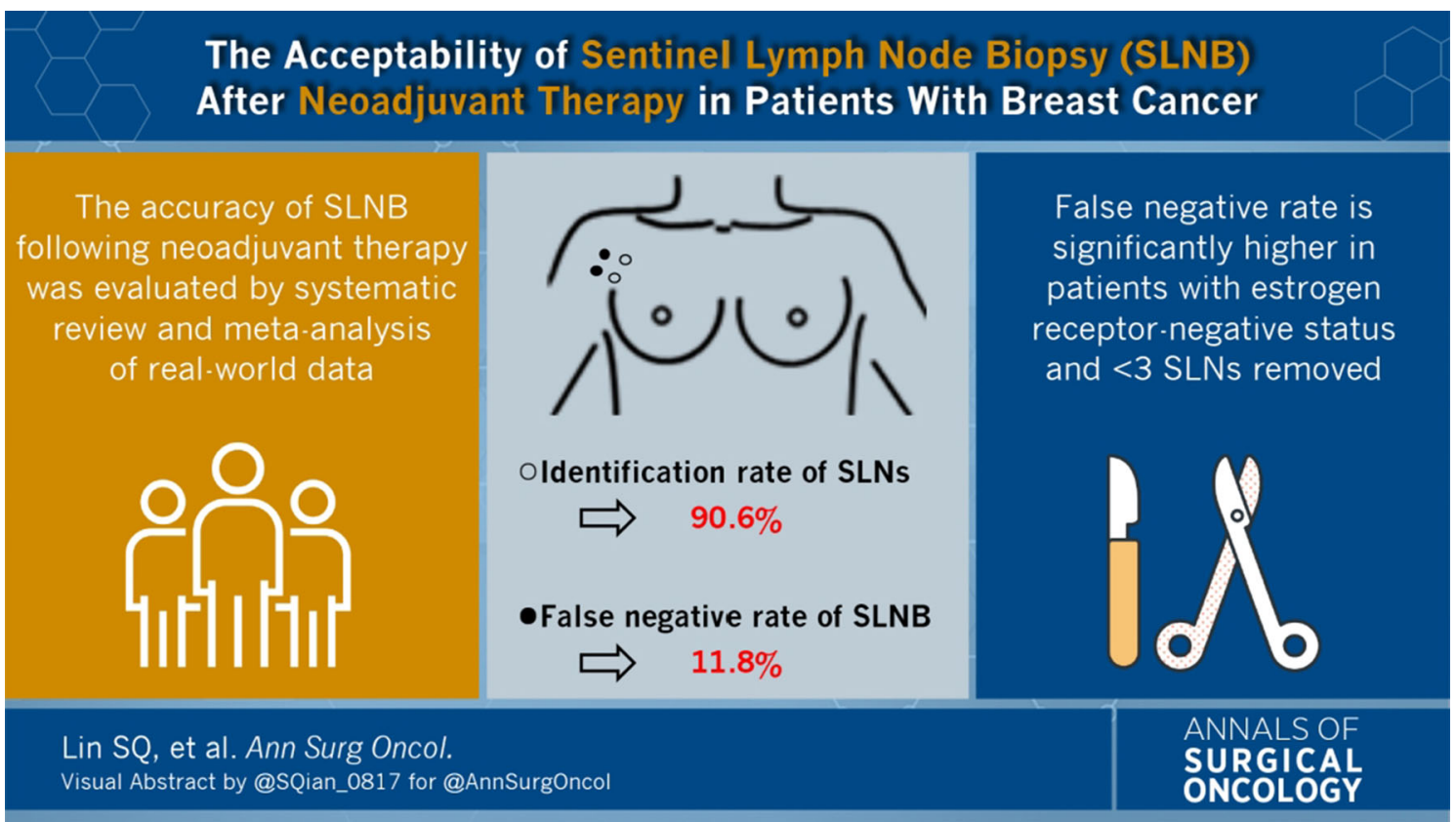

(C) Society of Surgical Oncology 2022

Publisher's Note Springer Nature remains neutral with regard to K.-W. Tam, MD, PhD

e-mail: kelvintam@h.tmu.edu.tw 